\title{
Prenatal VPA exposure and changes in sensory processing by the superior colliculus
}

\section{Georgia Dendrinos, Marie Hemelt and Asaf Keller*}

Program in Neuroscience, Department of Anatomy and Neurobiology, University of Maryland School of Medicine, Baltimore, MD, USA

Edited by:

John J. Foxe, Albert Einstein College of Medicine, USA

\section{Reviewed by:}

Albino J. Oliveira-Maia,

Champalimaud Foundation, Portugal

Adrian Rodriguez-Contreras, City

College of New York, USA

Manuel Gomez-Ramirez, The Johns

Hopkins University, USA

\section{*Correspondence:}

Asaf Keller, Department of Anatomy and Neurobiology, University of

Maryland School of Medicine,

20 Penn Street, Baltimore, MD 21201,

USA.

e-mail: kellerlab@gmail.com
Disorders involving dysfunctional sensory processing are characterized by an inability to filter sensory information, particularly simultaneously arriving multimodal inputs. We examined the effects of prenatal exposure to valproic acid (VPA), a teratogen linked to sensory dysfunction, on the behavior of juvenile and adult rats, and on the anatomy of the superior colliculus, a critical multisensory integration center in the brain. VPA-exposed rats showed deficits in colliculus-dependent behaviors including startle response, prepulse inhibition, and nociceptive responses. Some deficits reversed with age. Stereological analyses revealed that colliculi of VPA-treated rats had significantly fewer parvalbumin-positive neurons, a subset of GABAergic cells. These results suggest that prenatalVPA treatment affects the development of the superior colliculus and leads to persistent anatomical changes evidenced by aberrant behavior in tasks that require sensory processing.

Keywords: sensory processing, valproic acid, superior colliculus, rat

\section{INTRODUCTION}

Several neurocognitive disorders in humans involve dysfunctional sensory processing. These include but are not limited to autism spectrum disorders, attention deficit/hyperactivity disorders, fetal valproate syndrome, and putative sensory processing disorders. Sensory deficiencies in these disorders may manifest as hypo or hypersensitivity to sensory stimuli.

While the etiology of most of these disorders is unknown, fetal valproate syndrome is linked to embryologic insults with teratogens. Children exposed in utero to valproic acid (VPA) may present with a host of developmental and behavioral delays and physical malformations (Moore et al., 2000). Physical abnormalities range from severe to subtle and include spina bifida, heart defects, joint laxity, and facial dysmorphologies. Many exposed children lag in motor function and coordination, speech and learning. They often have decreased social interactions, hyperactivity, and difficulties with attention. Furthermore, humans exposed prenatally to VPA suffer from sensorimotor dysfunctions, most prominently hypersensitivity to innocuous sensory stimuli (Ardinger et al., 1988).

Similar sensory dysfunction can be reproduced in experimental animals, including rats, by administering VPA to the pregnant animal: offspring exhibit behavioral deficits that closely resemble those of humans with deficient sensory processing, including hypersensitivity to sensory stimuli (Rodier et al., 1997; Arndt et al., 2005; Schneider and Przewlocki, 2005; Markram et al., 2007; Markram and Markram, 2010). Thus, the VPA animal model offers an opportunity to explore mechanisms of teratogen-induced deficiencies in sensory processing.

Exposure to VPA likely affects a number of brain structures involved in sensory functions. Indeed, anatomical and electrophysiological abnormalities have been reported in a number of brain regions of adult rats following exposure to VPA in utero. These regions include the cerebral cortex, amygdala, cerebellum, and brainstem (Markram et al., 2007; Rinaldi et al., 2007, 2008b; Markram and Markram, 2010).

Another brain region presumably affected is the superior colliculus, which is critically involved in processing multisensory information (Stein and Meredith, 1993). Functioning to process and integrate visual, auditory, and somatosensory information and regulate motor acts, this structure is likely involved in the expression of the abnormalities inherent in disorders that involve sensory processing. For instance, animals exposed to VPA in utero display aberrant behaviors in auditory startle responses, sensorimotor gating, and nociceptive processing (Vorhees, 1987; Schneider and Przewlocki, 2005), behaviors that are mediated, at least in part, by the superior colliculus (Redgrave et al., 1996; Fendt, 1999; Fendt et al., 2001; Yeomans et al., 2006).

Here we test the hypothesis that prenatal VPA treatment alters the superior colliculus, resulting in sensorimotor deficits that persist into adulthood. We report that prenatal VPA treatment results in sensorimotor behavioral deficits and anatomical abnormalities in the superior colliculus.

\section{MATERIALS AND METHODS ANIMALS}

Female Sprague Dawley rats (Harlan) were mated overnight and vaginal lavage was used to test for the presence of sperm. The first day of gestation was designated as the day when the smear was sperm-positive. In some cases timed-pregnant females were used. Animals were exposed to a normal light/dark schedule. Females raised their own litters. Offspring were weaned on postnatal day 21 (PND 21). No more than three siblings of the same sex were housed together in cages. Control rats and VPA-treated rats were housed in separate cages. Animals had free access to food and water. All experiments were carried out according to IACUC regulations and federal guidelines. 


\section{VPA ADMINISTRATION}

Due to high rates of embryonic resorption and postnatal mortality [even beyond weaning (PND $21^{+}$)], several methods of VPA administration were employed. Table 1 summarizes various modes used, the timing of administration, and their success in terms of birth rate, litter size, adolescent survival rates, and sex ratios.

For injections, sodium valproate (Sigma-Aldrich, St. Louis, MO, USA) was dissolved in saline at a concentration of $250 \mathrm{mg} / \mathrm{ml}$ and the appropriate dose was administered intraperitoneally (ip). Experiments reported here were carried out on animals that received one of two VPA dosing schemes: (1) "single dose" (400$600 \mathrm{mg} / \mathrm{kg}$ ) of VPA or saline delivered on E12.5 or (2) "multiple doses" (200 mg/kg) of VPA or saline delivered on E11-13.

The first dosing scheme has been introduced by Rodier et al. (1996) and used in several subsequent studies (Rodier et al., 1997; Ingram et al., 2000; Schneider et al., 2001, 2006; Schneider and Przewlocki, 2005; Stodgell et al., 2006; Wagner et al., 2006; Rinaldi et al., 2008a,b). The second dosing scheme was included to resemble more clinically relevant, multiple exposures to VPA. Dosing schemes that consisted of more than 3 days of exposure, although higher in clinical relevance, resulted in high rates of mortality (data not shown) and were therefore not used.

Because offspring viability and sustainability of VPA-treated dams was of particular difficulty, it is possible that the rats that did survive prenatal exposure to VPA, and that were included in this study, had relatively mild consequences, and that the more severely affected died in utero or shortly after birth. The cohort of animals that survived may therefore not represent the severity of VPA's effects on sensory processing. Moreover, the consequences of fetal exposure to a teratogen are influenced by intrauterine position of the pups (Lipton et al., 1998), further increasing the variability within each litter.

\section{BEHAVIOR}

Behavioral experiments were carried out on offspring of VPAtreated and control animals that received either (1) a single i.p. injection of VPA ( 400 or $500 \mathrm{mg} / \mathrm{kg}$ ) or saline on E12.5 or (2) multiple day i.p. injections of VPA $(200 \mathrm{mg} / \mathrm{kg})$ or saline on E11-13.
Unless indicated, all behaviors were assessed in juveniles (PND 22-28) and adults (PND >60). Behavioral testing was run at the same time each day for each group of animals. Sample size for each individual behavioral test can be found in Tables 2 and 3 .

\section{ACOUSTIC STARTLE RESPONSE AND PREPULSE INHIBITION}

Acoustic startle response was measured using San Diego Instruments SR-LAB ${ }^{\circledR}$ Startle Response System (San Diego, CA, USA). Animals were tested in a ventilated enclosure mounted on a Plexiglas frame. Motion was detected and transduced by a piezoelectric sensor below the Plexiglas frame. Subjects acclimated to the testing chamber for 5 min during which only background noise ( $68 \mathrm{~dB})$ was delivered. Acoustic stimuli of white noise between 69 and $113 \mathrm{~dB}$ were randomly presented for a duration of $40 \mathrm{~ms}$ each. Inter-trial intervals ranged between 5 and $10 \mathrm{~s}$. Testing lasted approximately $20 \mathrm{~min}$, during which the maximal velocity of the animals' startle response was measured. This metric, referred to as startle amplitude, is measured in arbitrary units.

Prepulse inhibition (PPI) was measured using the same apparatus. Both prepulse and pulse stimuli consisted of white noise which were generated by the testing apparatus. Background level was set at $68 \mathrm{~dB}$, prepulse level at $80 \mathrm{~dB}$ and stimulus duration was $20 \mathrm{~ms}$. Pulse level was $120 \mathrm{~dB}$ and duration was $40 \mathrm{~ms}$, the interval between prepulse and pulse was $100 \mathrm{~ms}$ and inter-trial interval varied between 10 and $15 \mathrm{~s}$. Percentage of PPI was calculated as: (amplitude of startle response) - (amplitude of prepulse response)/(amplitude of startle response) $\times 100$.

\section{MECHANICAL NOCICEPTION}

Von Frey rigid filaments were used to assess mechanical withdrawal response probability. Rats were gently restrained by hand during testing. Graded filaments of increasing force were applied to the dorsal surface of the hindpaw or to the vibrissa pad until bending to deliver calibrated force ranging from 0.2 to $180.0 \mathrm{~g}$. Response probability was calculated as number of times out of five the subject withdrew or vocalized.

\section{THERMAL NOCICEPTION}

Thermal pain threshold was determined using the Hargreaves method (Hargreaves et al., 1988). A radiant heat stimulus of $33^{\circ} \mathrm{C}$

Table 1 | Summary of success of VPA administration paradigms.

\begin{tabular}{|c|c|c|c|c|c|c|}
\hline VPA treatment & $\begin{array}{l}\text { Day(s) of } \\
\text { treatment }\end{array}$ & $\begin{array}{l}\% \text { Of females that } \\
\text { gave birth }\end{array}$ & $\begin{array}{l}\text { Litter size } \\
\text { (average) }\end{array}$ & $\begin{array}{l}\% \text { Of pups that } \\
\text { reach PND } 21\end{array}$ & $\begin{array}{l}\text { Total viable } \\
\text { offspring (average) }\end{array}$ & $\begin{array}{l}\text { Offspring male: } \\
\text { female ratio }\end{array}$ \\
\hline \multicolumn{7}{|l|}{$600 \mathrm{mg} / \mathrm{kg}$} \\
\hline VPA & E12.5 & 57 & 9 & 50 & 4.5 & $1.5: 1$ \\
\hline Saline & & 88 & 10.3 & 69 & 7.1 & $1: 1.5$ \\
\hline \multicolumn{7}{|l|}{$500 \mathrm{mg} / \mathrm{kg}$} \\
\hline VPA & E12.5 & 42 & 5.2 & 50 & 2.6 & $0.7 / 1$ \\
\hline Saline & & 88 & 10.3 & 69 & 7.1 & $0.8 / 1$ \\
\hline \multicolumn{7}{|l|}{$400 \mathrm{mg} / \mathrm{kg}$} \\
\hline VPA & E12.5 & 100 & 7.7 & 74 & 5.7 & $1: 2$ \\
\hline Saline & & 88 & 10.3 & 69 & 7.1 & $0.8 / 1$ \\
\hline \multicolumn{7}{|l|}{$200 \mathrm{mg} / \mathrm{kg}$} \\
\hline VPA & E11-13 & 100 & 12.8 & 92 & 11.8 & $1.1 / 1$ \\
\hline Saline & & 100 & 11 & 95 & 10.5 & $1.1 / 1$ \\
\hline
\end{tabular}


Table 2 | Summary of behavioral results for groups receiving a single dose of VPA or saline.

\begin{tabular}{|c|c|c|c|c|c|}
\hline & VPA on E12.5 & $n$ & Control 12.5 & $n$ & $p$ Value \\
\hline \multicolumn{6}{|l|}{ PPI\% } \\
\hline Juvenile & $3.9 \pm 5.6$ & 29 & $20.7 \pm 4.9$ & 18 & 0.03 \\
\hline \multicolumn{6}{|c|}{ von FREY FACE (g) 5/5 } \\
\hline Juvenile & $86.3 \pm 6.0$ & 56 & $111 \pm 8.2$ & 26 & 0.005 \\
\hline Juvenile & $31 \pm 2.2$ & 56 & $46 \pm 3.3$ & 26 & 0.0002 \\
\hline Adult & $140 \pm 19.7$ & 16 & $167 \pm 13.3$ & 6 & 0.21 \\
\hline \multicolumn{6}{|c|}{ THERMAL LATENCY (s) } \\
\hline Juvenile & $\mathrm{n} / \mathrm{a}$ & $\mathrm{n} / \mathrm{a}$ & $\mathrm{n} / \mathrm{a}$ & $\mathrm{n} / \mathrm{a}$ & $\mathrm{n} / \mathrm{a}$ \\
\hline Adult & $11.8 \pm 0.7$ & 16 & $8.2 \pm 0.5$ & 6 & 0.003 \\
\hline \multicolumn{6}{|c|}{ EXPLORATORY DISTANCE (ARBITRARY UNITS) } \\
\hline Juvenile & $2225 \pm 62$ & 27 & $1793 \pm 111$ & 18 & 0.001 \\
\hline Adult & $4691 \pm 259$ & 8 & $4982 \pm 482$ & 3 & 0.54 \\
\hline
\end{tabular}

Data expressed as mean \pm SEM.

Table 3 | Summary of behavioral results for groups receiving multiple doses of VPA or saline.

\begin{tabular}{|c|c|c|c|c|c|}
\hline & VPA on E11-13 & $n$ & Control 11-13 & $n$ & $p$ Value \\
\hline \multicolumn{6}{|l|}{ PPI\% } \\
\hline Juvenile & $18.1 \pm 3.6$ & 34 & $28.9 \pm 5.4$ & 21 & 0.12 \\
\hline \multicolumn{6}{|c|}{ von FREY FACE (g) 5/5 } \\
\hline Juvenile & $84.0 \pm 4.1$ & 68 & $98 \pm 6.0$ & 40 & 0.04 \\
\hline Juvenile & $39 \pm 2.2$ & 68 & $42 \pm 2.7$ & 42 & 0.21 \\
\hline adult & $165 \pm 13.7$ & 26 & $142 \pm 11.3$ & 22 & 0.11 \\
\hline \multicolumn{6}{|c|}{ THERMAL LATENCY (s) } \\
\hline Juvenile & $\mathrm{n} / \mathrm{a}$ & $\mathrm{n} / \mathrm{a}$ & $\mathrm{n} / \mathrm{a}$ & $\mathrm{n} / \mathrm{a}$ & $\mathrm{n} / \mathrm{a}$ \\
\hline Adult & $11.7 \pm 0.5$ & 26 & $9.0 \pm 0.5$ & 20 & 0.0003 \\
\hline Juvenile & $1828 \pm 125$ & 23 & $2203 \pm 111$ & 21 & 0.06 \\
\hline Adult & $4798 \pm 125$ & 13 & $4483 \pm 162$ & 11 & 0.11 \\
\hline
\end{tabular}

Data expressed as mean \pm SEM.

(IITC, Woodland Hills, CA, USA) was applied to the plantar surface of the rat's hindpaw until it was withdrawn. A cutoff time of $20 \mathrm{~s}$ was used to prevent injury. The test was repeated three times for each hindpaw. Data are reported as the average (of three trials) latency to paw withdrawal. To prevent sensitization, at least $5 \mathrm{~min}$ separated each trial.

\section{OPEN FIELD BEHAVIOR AND SOCIABILITY}

A three-compartment chamber (dimensions: $90 \mathrm{~cm} \times 50 \mathrm{~cm} \times$ $32 \mathrm{~cm}$ ) made of black plastic was used to assess open field and sociability (Crawley, 2004). For open field behavior subjects were placed in the middle chamber and a sliding door revealed two chambers for the test rat to freely explore. Rats 
were video recorded for $5 \mathrm{~min}$ and their behavior was analyzed using Topscan (CleverSys, Reston, VA, USA) software. The distance (arbitrary units) traveled while exploring was used for data analysis.

The same apparatus was used to assess sociability. Subjects were placed in the middle (neutral) chamber and a sliding door revealed two chambers (social and non-social) to the test rat. Each of the two chambers held a clear perforated plexiglas sub-chamber (dimensions: $20 \mathrm{~cm} \times 20 \mathrm{~cm} \times 20 \mathrm{~cm}$ ) into which an age-matched and sex-matched stranger rat was placed. The side with the subchamber housing the stranger rat was designated as the "social" chamber. Rats were video recorded for $5 \mathrm{~min}$ and their behavior was analyzed using Topscan software. Time spent in each chamber was used for data analysis. The order of running the open field and sociability tests was counterbalanced for each group.

\section{ANATOMY}

All anatomical experiments were carried out on adult (PND >60) offspring of animals that received, on E12.5, a single $600 \mathrm{mg} / \mathrm{kg}$ i.p. injection of VPA or saline. See Figure $\mathbf{5}$ for sample size for each stereological analysis.

\section{IMMUNOHISTOCHEMISTRY}

Rats were perfused transcardially with $4 \%$ paraformaldehyde, brains were removed, placed in fixative overnight and equilibrated in a $30 \%$ sucrose solution. Fifty Micrometer thick coronal sections were cut on a freezing stage microtome or cryostat from Bregma -8.0 to $-5.6 \mathrm{~mm}$ (Paxinos and Watson, 1998). Sections were washed with PBS and incubated in mouse antiparvalbumin (1:75,000; Swant, Bellinzona, Switzerland) or mouse anti-NeuN (1:120,000, Sigma-Aldrich, St. Louis, MO, USA) and $0.4 \%$ Triton-X PBS for $48 \mathrm{~h}$ at room temperature. After washing, sections were incubated for $1 \mathrm{~h}$ in biotinylated rabbit anti-mouse (1:600; Jackson ImmunoResearch, West Grove, PA, USA) and $0.4 \%$ triton-X PBS. After PBS rinses, sections were incubated for $30 \mathrm{~min}$ in $\mathrm{ABC}$ elite solution (Vector Laboratories, Burlingame, CA, USA) and the reaction product developed using nickelDAB. Sections were air dried overnight, mounted, dehydrated, and cover-slipped in DPX. Neutral red (1\%) was used for Nissl staining.

\section{DOUBLE IMMUNOFLUORESCENCE}

Rats were transcardially perfused with $4 \%$ paraformaldehyde, brains removed and placed in fixative overnight and placed in cryoprotectant for 1 week. After gelatin embedding, $50 \mu \mathrm{m}$ thick coronal sections were cut on a cryostat from Bregma -8.0 to $-5.6 \mathrm{~mm}$, washed with PBS and incubated in blocking solution ( $2 \%$ BSA in $0.1 \%$ Triton in $0.05 \mathrm{M}$ PBS) for $1 \mathrm{~h}$. Sections were then incubated for $48 \mathrm{~h}$ at room temperature in primary antibody, rabbit anti-GABA (1:100,000; Sigma-Aldrich), or mouse anti-parvalbumin (1:60,000; Swant). After washing, sections were incubated for $2 \mathrm{~h}$ at room temperature in secondary antibody tagged with Cy3 or Cy2 (1:1000; Jackson ImmunoResearch). Slices were dehydrated and mounted using DPX. Tissue was visualized using an Olympus (Center Valley, PA, USA) Fluoview confocal scanning system microscope.

\section{STEREOLOGY}

StereoInvestigator's (MBF Biosciences, Williston, VT, USA) optical fractionator probe was used to obtain unbiased estimates of cell number, cell density, and area volume. Counting frames were $50 \mu \mathrm{m}$ by $50 \mu \mathrm{m}$; grid size was $305 \mu \mathrm{m}$ by $175 \mu \mathrm{m}$; guard zones were $5 \mu \mathrm{m}$; and dissector height was $15 \mu \mathrm{m}$. Whereas the borders of the superficial layer of the superior colliculus are distinct, the borders of the intermediate and deep layers are not. To circumvent any ambiguity, we defined the lateral border of these regions as a transverse line extending from the lateral edge of the superficial layer to the lateral most border of the periaqueductal gray (PAG; see Figure 1).

\section{Statistical analyses}

For behavioral analyses, data collected from the single dose VPA group was compared to the single dose saline group. Similarly, data collected from the multiple dose VPA group was compared to the multiple dose saline group. In most cases, comparisons were made using the Mann-Whitney $U$ non-parametric test for independent samples. Startle responses, however, were tested using a repeated-measures ANOVA with Bonferroni correction for multiple comparisons to determine significance at individual decibel levels.

For anatomical studies, we compared animals that received a single dose of VPA $(600 \mathrm{mg} / \mathrm{kg})$ at $\mathrm{E} 12.5$ to controls that

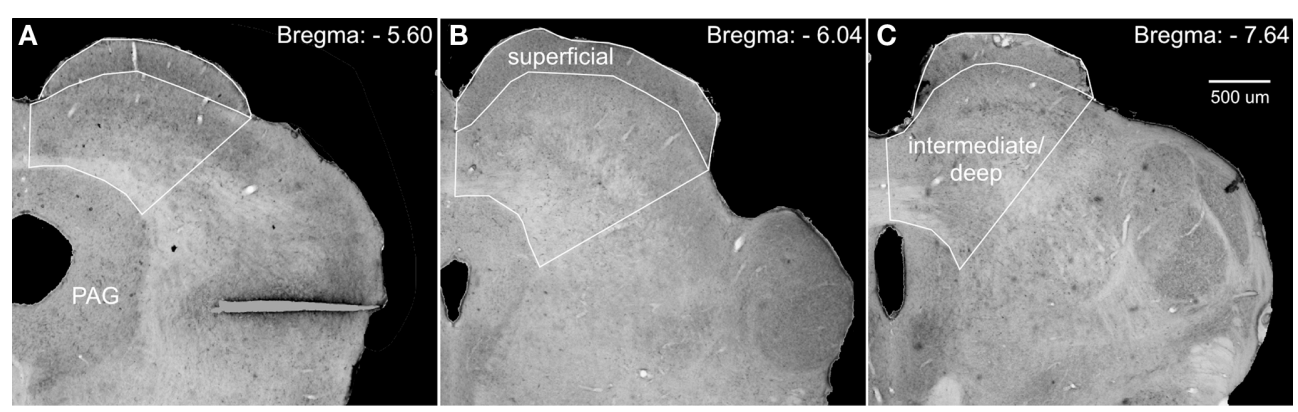

FIGURE 1 | Photomicrographs of Nissl-stained coronal sections through the superior colliculus, depicting the regions used for stereological analyses. We defined the lateral border of the intermediate/deep layers of the superior colliculus by a line extending from the lateral edge of the superficial layers to the lateral border of the periaqueductal gray (PAG). (A-C) Show increasingly more posterior sections and subsequently adjusted borders. 
received saline at E12.5. All comparisons were made using the Mann-Whitney $U$ non-parametric test for independent samples.

\section{RESULTS}

\section{BEHAVIOR}

Previous studies have shown that the effects of prenatal exposure to VPA on certain behavioral measures are correlated with the postnatal age at which these behaviors are quantified (Markram et al., 2007; Markram and Markram, 2010, \#68472). We therefore analyzed our experimental and control groups in a number of tests, administered when they were juveniles (PND 21-30) and adults (PND >60). We used two dosing paradigms: a single dose delivered at E12.5 and multiple doses delivered daily on E11-13 (see Materials and Methods). Tables 2 and 3 summarize these findings.

\section{STARTLE RESPONSE}

A common finding in subjects with sensory processing disturbances is an abnormal response to auditory stimuli (Ayres, 1979), which can be measured by the startle response. The acoustic startle response quantifies the velocity of movement in response to a sudden sound. The startle response of juvenile rats exposed in utero to a single dose of VPA $(n=29)$ was significantly different $(p s<0.05$, one-way ANOVA, Bonferroni post hoc) than that of age-matched controls for several decibel levels ( $n=18$; Figure 2A). Rats treated with a multiple injections of VPA responded similarly to controls except for a significantly lowered startle response at $97 \mathrm{~dB}$ (Figure 2C). By contrast, in adults there were no significant differences in the startle responses for VPA-treated rats that received either a single injection of VPA $(n=8)$ or multiple injections of
A

Juvenile acoustic startle response

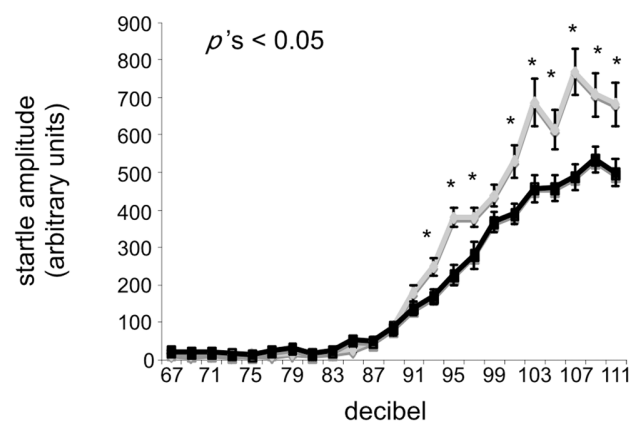

C

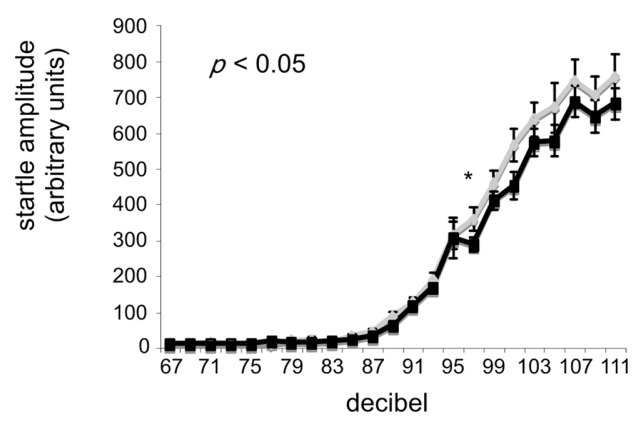

E

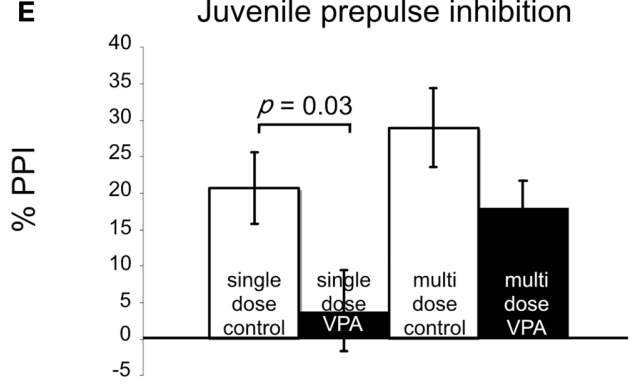

FIGURE 2 | VPA-treated rats exhibit abnormal startle responses and prepulse inhibition. Acoustic startle response of animals receiving a single dose $(\mathbf{A}, \mathbf{B})$ or multiple doses of VPA $\mathbf{( C , D )}$. (A) Juvenile rats receiving a single dose of VPA show decreased responses to high intensity acoustic stimuli. (B) These changes are reversed in adult rats. Juvenile (C) and adult (D) rats
B

Adult acoustic startle response

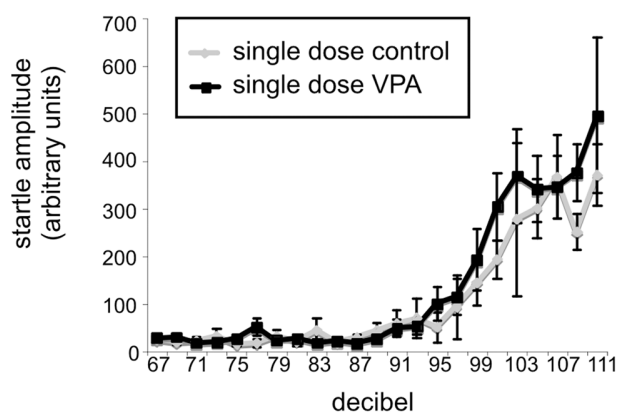

D

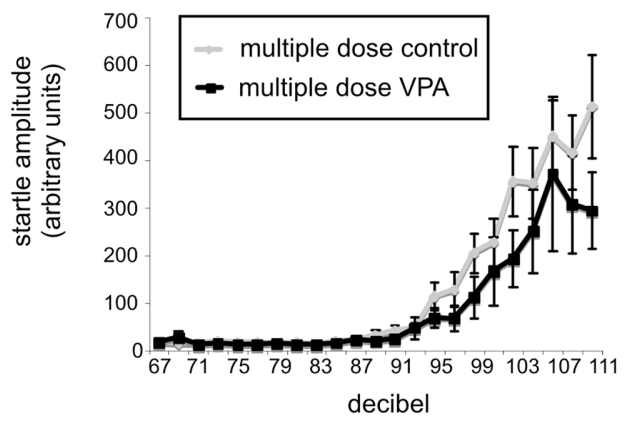

$\mathbf{F}$

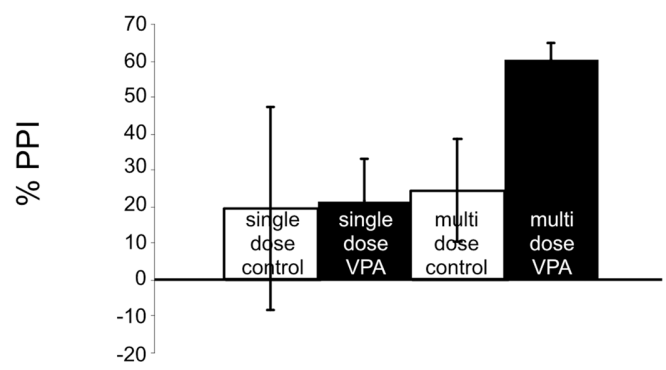

treated with multiple doses of VPA show no difference from controls. (E,F). Juvenile and adult prepulse inhibition. (E) Juvenile rats treated with a single dose of VPA show decreased prepulse inhibition. (F) This is reversed in adult rats. Juvenile and adult rats treated with multiple doses of VPA respond similarly to controls. 
VPA ( $n=13$; Figures $2 \mathbf{B}, \mathbf{D}$ ) when compared to their respective controls.

\section{PREPULSE INHIBITION}

Sensorimotor gating is also commonly abnormal in subjects with sensory processing disturbances, including animals and humans exposed to VPA in utero (Ardinger et al., 1988; Rogers and Ozonoff, 2005; Schneider and Przewlocki, 2005). We assessed sensorimotor gating with a PPI paradigm. This paradigm quantifies the suppressive effects of a non-startling, antecedent auditory stimulus on motor responses to a startling stimulus. Juvenile rats treated in utero with a single dose of VPA $(n=29)$ exhibited significantly decreased PPI compared to control rats $(n=18$; $p=0.03$, MannWhitney $U$; Figure 2E) whereas juvenile rats treated with multiple doses of VPA did not. In adult rats treated with either a single dose $(n=8)$ of VPA or multiple doses of VPA $(n=13)$, PPI was not significantly different $(p=1.00$ or 0.11 , respectively, MannWhitney $U$ ) than that of their age-matched respective control groups (Figure 2F).

\section{MECHANICAL AND THERMAL NOCICEPTION}

Tactile perception and the perception of pain are commonly affected in disorders involving disturbed sensory processing (BarShalita et al., 2009). We assessed mechanical nociceptive responses with calibrated von Frey filaments applied either to the face or hindpaw.

When stimuli were applied to the face, juvenile rats treated with a single dose of VPA ( $n=56$ vibrissae pads) showed a significant decrease in threshold ( $p=0.005$, Mann-Whitney $U$, Figure $3 \mathbf{A}$ ) when compared to controls $(n=26)$. Juvenile rats treated with multiple doses of VPA $(n=68)$ also showed lowered thresholds to mechanical stimuli in comparison to control rats ( $n=40, p=0.04$, Mann-Whitney $U$, Figure 3C). When stimuli were applied to the hindpaw, only juvenile rats treated with a single dose of VPA $(n=56)$ exhibited a significant decrease in threshold ( $p=0.0002$, Mann-Whitney $U$, Figure 3E), whereas the multiple dose paradigm of VPA $(n=42)$ had no significant effect (Figure 3G).

In response to stimuli applied to the face, adult rats treated with a single dose of VPA $(n=16)$ or multiple doses of VPA $(n=26)$ showed similar response probability to their respective control groups (Figures 3B,D). Similarly, for stimuli applied to the hindpaw, neither group of adult rats differed significantly from controls (Figures 3F,H).

Another type of perception that is affected in disorders with disrupted sensory processing disorders is that of thermal pain (Bar-Shalita et al., 2009). We determined thermal response thresholds using the Hargreaves method (Hargreaves et al., 1988) in which a heat stimulus is applied to the plantar surface of the rat's hindpaw until the paw is withdrawn. Paw withdrawal latencies in adult rats treated with either a single $(n=16)$ or multiple doses of VPA $(n=26)$ were significantly higher $(p=0.003$, and $p=0.0003$, respectively, Mann-Whitney $U$ ) compared to controls (Figure 3I). No data were collected for thermal latency of juvenile VPA-treated or age-matched control rats.

\section{SOCIABILITY}

Subjects with disorders involving disturbed sensory processing often exhibit abnormal social interaction (Ayres, 1979). We assessed sociability using an established paradigm (Crawley, 2004) in which we monitored the behavior of a freely moving test rat in a multiple-compartment chamber in which an age and sex-matched "stranger" rat is restricted to one enclosed compartment (see Materials and Methods). Juvenile rats treated with a single dose of VPA $(n=29)$, but not those treated with multiple doses, spent significantly less time $(p=0.03$, Mann-Whitney $U$ ) with a novel rat when compared to controls (Figure 4A). Adult rats treated with either a single dose $(n=8)$ or multiple doses $(n=13)$ of VPA also did not differ from controls (Figure 4B) in the amount of time they spent with a novel rat.

\section{EXPLORATORY BEHAVIOR}

Preceding open field testing in juveniles revealed significantly more exploratory behavior from juveniles treated with a single dose of VPA ( $n=27, p=0.003$, Mann-Whitney $U$; Figure 4C). However, there were no exploratory differences between juvenile rats treated with multiple doses of VPA or either group of adult rats (Figure 4D) and their respective control groups.

\section{COLLICULAR ANATOMY}

The effects of VPA administration on a number of behaviors related to sensory processing suggest that prenatal VPA administration results in abnormal development of related brain structures, including the superior colliculus.

We tested this hypothesis by analyzing the superior colliculi of adult rats, with the use of stereological procedures (see Materials and Methods). First, we compared the total number of Nissl-stained cells in colliculi of animals treated with single dose of VPA $(600 \mathrm{mg} / \mathrm{kg}$ on E12.5; $n=10)$ with agematched controls $(n=10)$. There were no significant differences in the total number of cells in the superficial layers $(p=0.26$, Mann-Whitney $U$ ). Control animals had 385,100 $\pm 34,000$ cells whereas VPA-treated animals had $314,700 \pm 32,000$ cells. Likewise, the intermediate/deep showed no difference in Nissl cell number $(p=0.45$, Mann-Whitney $U$; control: $745,600 \pm 60,000$; VPA: $644,300 \pm 64,400$ ) layers (Figures 5D,E). There was also no significant difference in volume between treatment and control groups $(p=0.71$, Mann-Whitney $U$, for superficial and intermediate/deep layers, respectively). Analysis of the number of neurons, determined from sections labeled with the neuron-specific marker NeuN, also revealed no effects of VPA treatment on the number of neurons in the superficial $(p=1.0$, Mann-Whitney $U)$ or intermediate/deep $(p=1.0$, Mann-Whitney $U$ ) layers of the superior colliculus. Control animals $(n=4)$ had $246,300 \pm 37,900$ neurons whereas VPAtreated animals $(n=4)$ had $260,000 \pm 5000$ neurons in the superficial layer. In the intermediate/deep layers, control animals had 353,000 \pm 51000 neurons and VPA-treated animals had 366,600 $\pm 15,700$ neurons. Volumetric analysis also revealed no significant differences between VPA-treated and control rats ( $p=1.00$, Mann-Whitney $U$, for both superficial and intermediate/deep layers).

We reasoned that some of the behavioral effects of VPA might reflect abnormal development of inhibitory neurons in the superior colliculus. We focused our analysis on inhibitory neurons that express parvalbumin because these neurons comprise a large and potent population of GABAergic neurons in various brain 

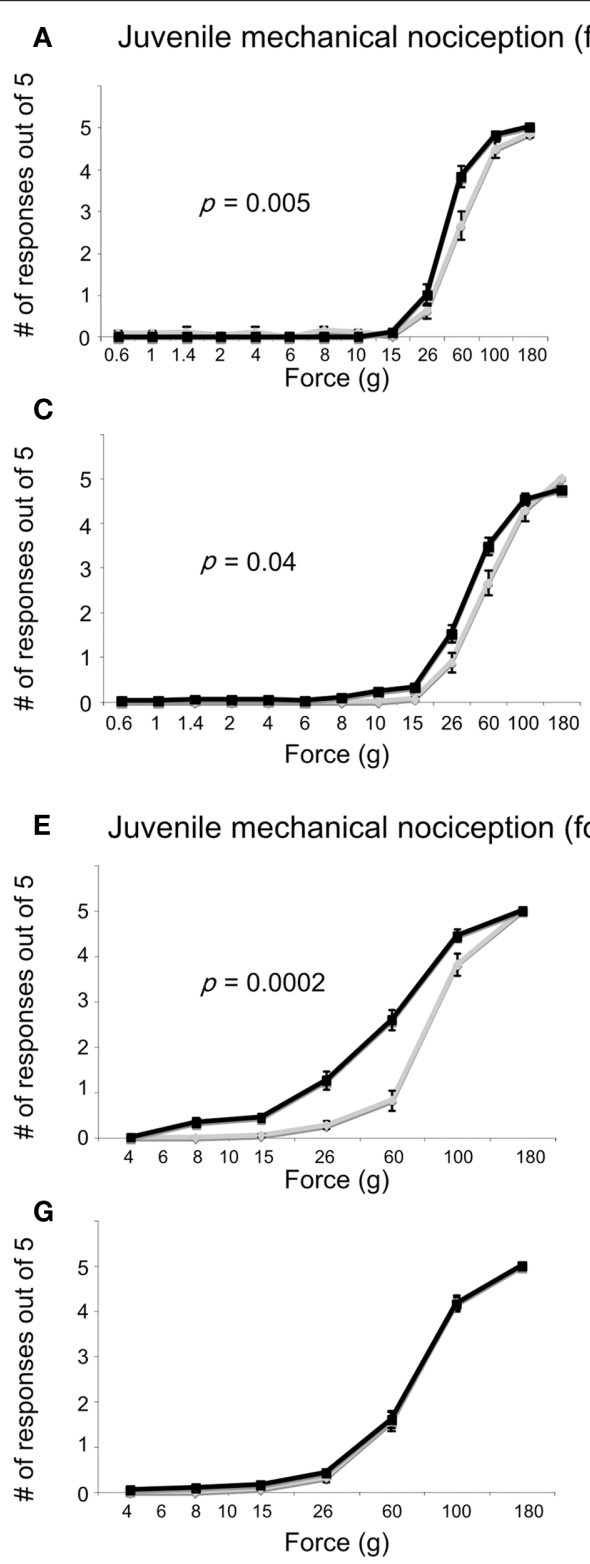

FIGURE 3 | Effect of VPA treatment on nociceptive behavior. (A-D). Stimulus response curves to mechanical stimulation of the vibrissae pad. Juvenile rats treated with a single dose of VPA

(A) show increased sensitivity to mechanical stimuli. (B) This effect was reversed in adults. Juvenile (C) and adult (D) rats treated with multiple doses of VPA show increased sensitivity to mechanical stimuli applied to the face.
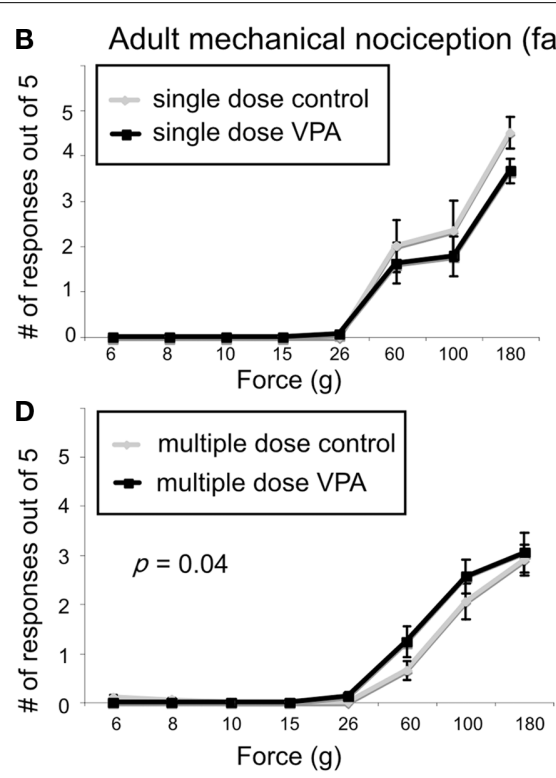

F Adult mechanical nociception (foot)
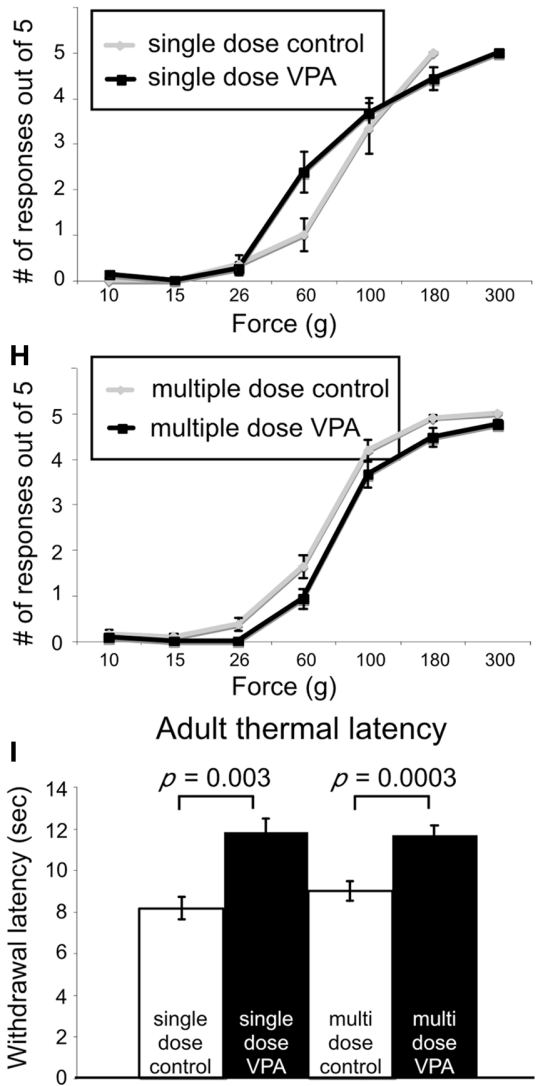

(E-F) Stimulus response curves to mechanical stimulation of the hindpaw. (E) Juvenile rats treated with a single dose of VPA show increased sensitivity to mechanical stimuli. (F) This is reversed in adults. (G,H) Juvenile and adult rats treated with multiple doses of VPA respond similarly to controls. (I) VPA-treated rats show decreased sensitivity to thermal noxious stimulus. 


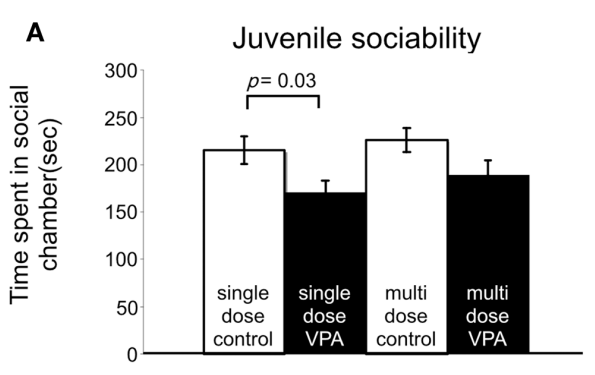

C Juvenile exploratory behavior

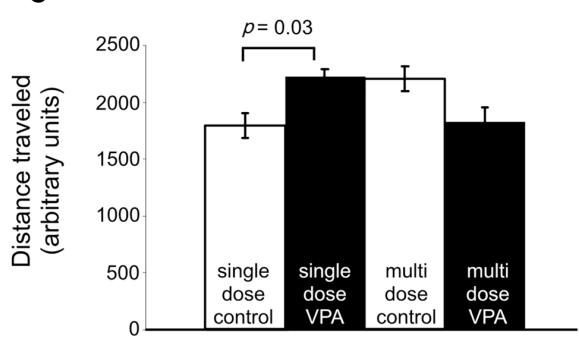

FIGURE 4 | Effect of VPA treatment on sociability and

exploratory behavior. (A) Juvenile rats treated with a single dose of VPA spend less time with a novel rat. (B) This effect is reversed in adults. Juvenile and adult rats treated with multiple doses of VPA

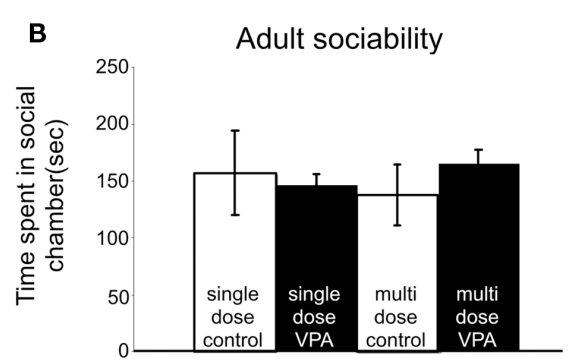

D Adult exploratory behavior

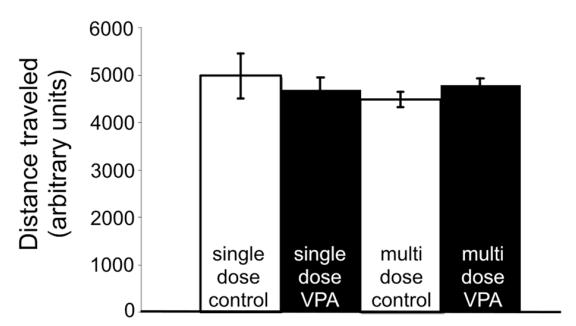

respond similarly to controls. (C) Juvenile rats treated with a single dose of VPA explore more than controls. (D) This is reversed in adults. Juvenile and adult rats treated with multiple doses of VPA respond similarly to controls. regions, including the colliculus (Monyer and Markram, 2004). We confirmed that parvalbumin is a reliable marker of GABAergic neurons in the colliculus by double labeling for parvalbumin and GABA (see Materials and Methods; Figures 5A-C). Quantitative analysis (six animals, 64 sections, 915 cells) revealed that $95 \%$ of the neurons labeled for parvalbumin were also stained with the GABA antibody. This finding indicates that parvalbumin is a reliable marker of inhibitory neurons in the rat superior colliculus.

Stereological analysis of sections stained for parvalbumin revealed that, compared to controls $(n=10)$, there are about $30 \%$ fewer parvalbumin-positive neurons in VPA-treated rats $(n=10)$, both in the superficial ( $p=0.04$, Mann-Whitney $U$ ) and intermediate/deep layers $(p=0.003$, Mann-Whitney $U$; Figures 5F,G). In the superficial layer, adult rats treated with a single dose of VPA had an average of 19,000 $\pm 5,000$ parvalbumin-positive neurons whereas control rats had $26,000 \pm 11,000$. In the intermediate/deep layers, the average number of parvalbumin-positive cells was $14,000 \pm 4,000$ in rats that were treated with a single dose of VPA and 21,000 \pm 5000 in controls. There was no significant difference in volume between control and treatment groups $(p=0.60$ and $p=0.92$, Mann-Whitney $U$ for superficial and intermediate/deep layers, respectively). No data were collected from adult rats treated with multiple doses of VPA or from any juvenile rats.

\section{DISCUSSION}

Previous studies demonstrated that prenatal exposure to VPA is teratogenic, resulting not only in gross physical deformations but also producing behavioral abnormalities (Arpino et al., 2000; Moore et al., 2000; Alsdorf and Wyszynski, 2005; Arndt et al., 2005; Wyszynski et al., 2005). The current study provides evidence that
VPA also has detrimental effects on sensory processing. This is manifested as behavioral deficits in sensorimotor tasks, as well as anatomical abnormalities of the superior colliculus, a structure known to be involved in sensory processing. These deficits were typically present in animals that received a single dose of VPA on E12.5, and most reversed with age.

In most instances, a single dose of VPA at E12.5 (400 or $500 \mathrm{mg} / \mathrm{kg}$ ) resulted in more severe behavioral deficits than multiple doses of VPA delivered on E11-13 (200 mg/kg). This may indicate that a minimum dose, precise timing of exposure, or the concurrence of these factors are necessary to precipitate the effects shown in this study. As the pharmacokinetics of VPA suggest that it is a quickly metabolized drug (half life $\sim 2.3 \mathrm{~h}$; Binkerd et al., 1988), it is highly unlikely that plasma concentrations of VPA would be additive over consecutive days. Therefore, it would appear that prenatal exposure to a dose exceeding $200 \mathrm{mg} / \mathrm{kg}$ on at least E12.5 of gestation is minimally required to elicit behaviors indicative of dysfunctional sensory processing.

\section{BEHAVIORAL FINDINGS}

\section{Startle response and prepulse inhibition}

We report that juveniles treated with a single dose of VPA startle significantly less to an unexpected acoustic stimulus across a range of decibel levels. Our findings are consistent with previous studies demonstrating that in utero intra-peritoneal VPA treatment $(500-600 \mathrm{mg} / \mathrm{kg}$ ) has no effect on startle responses of adult rats (Schneider and Przewlocki, 2005; Markram et al., 2007). By contrast, long-term treatment of dams with VPA by gavage is reported to suppress the startle response of adult rats (Vorhees, 1987). To our knowledge, ours is the first study to test the startle response in VPA-treated juvenile rats. 


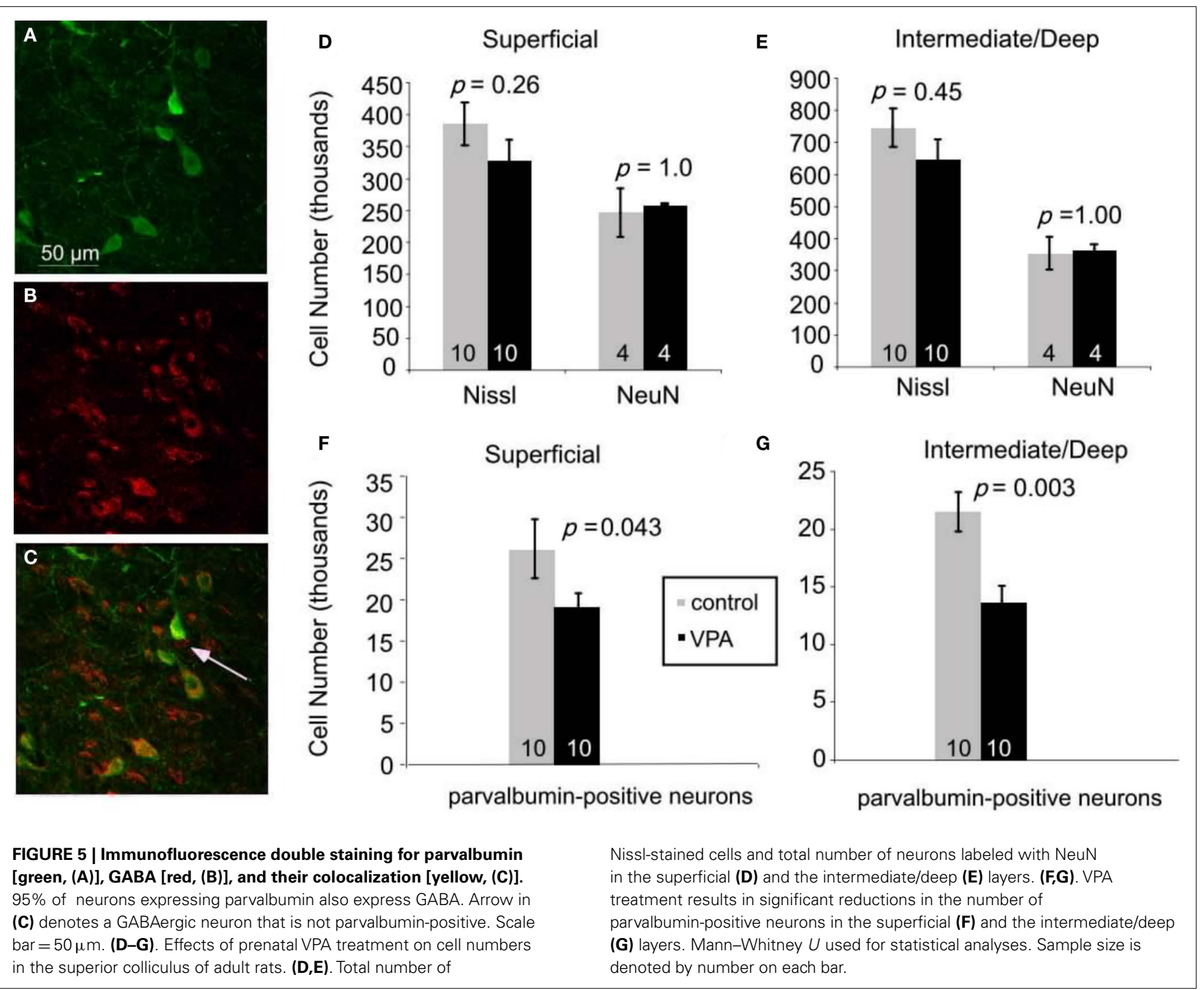

Our data show that juveniles treated with a single dose of VPA show a decrease in PPI. We did not observe any significant differences in PPI of adult rats. Previous studies reported that treatment with higher doses of VPA $(\geq 500 \mathrm{mg} / \mathrm{kg})$ result in reductions in PPI in adult rats (Schneider and Przewlocki, 2005; Markram et al., 2007). It is possible that these results differ from ours because of differences in the PPI protocol (we used louder pre-pulses and longer inter-trial intervals) or to differences in VPA concentrations. We are not aware of similar studies in juvenile, VPA-treated rats.

\section{Mechanical and thermal nociception}

We found that juveniles treated with a single dose of VPA show hyperalgesia to mechanical stimuli applied to the face or the hindpaw. Consistent with our findings, Schneider and Przewlocki (2005) report that single dose VPA effects on mechanical response probability are age-dependent, such that young adults (PND 30-50) treated prenatally with VPA have decreased thresholds, but older adults (PND 90-120) do not. These studies were conducted only on the hindpaw of the rat. To our knowledge, similar studies have not been carried out in juvenile rats treated with VPA or on any rats assessing mechanical response probability of the face.

Our data also show that adult rats treated with either a single dose or multiple doses of VPA have take longer to withdraw their paws from a radiant heat source. These results agree with previous studies showing that adult VPA-treated rats exhibit increased latency to withdrawing from a thermal stimulus (Schneider et al., 2001; Schneider and Przewlocki, 2005; Markram et al., 2007). These data suggest that VPA-treated rats may be less sensitive to thermal stimuli than mechanical stimuli, possibly due to VPA's distinct mechanism of action on different somatosensory receptors.

\section{Sociability and exploratory behavior}

We report that juvenile rats treated with a single dose of VPA explore more than controls in an open field but spend less time in a social chamber with a stranger rat. This strongly suggests that our results reflect an aversion to exploring a social environment and not a new environment in general. We did not find any differences in exploratory behavior or sociability in adults treated with VPA. Previous studies in which sociability was evaluated by 
monitoring pinning behavior and anogenital inspection report that adult rats treated with VPA are socially impaired (Schneider and Przewlocki, 2005; Markram et al., 2007). We are not aware of other studies using VPA in which adult sociability was measured using the approach we used, nor are we aware of studies on the effects of VPA on sociability in juvenile rats.

\section{ANATOMICAL FINDINGS}

Our data show that adults treated with a single dose of VPA are not statistically different from controls in terms of total cell and total neuronal number in the superior colliculus. However, VPA-treated rats show significant reductions in the number of parvalbuminpositive neurons. Such a decrease in interneuron number leads to a change in the synaptic ratio between excitation and inhibition in this structure.

Disruption in excitation/inhibition balance has long been linked to behavioral abnormalities and is implicated in numerous psychiatric disorders such as schizophrenia, anxiety, attention disorders, and autism (Truitt et al., 2007; Gruber et al., 2010; Won et al., 2011). It is therefore likely that a perturbation of this balance within the colliculus, such as a change in inhibitory interneurons, may result in abnormal behaviors in colliculus-dependent behaviors.

While all immunohistochemical findings are subject to how well an antigen can be detected by a particular antibody, it is also important to note that these stereological results yield from VPAtreated animals that received $600 \mathrm{mg} / \mathrm{kg}$ of VPA on E12.5. Our behavioral studies, however, used rats treated with lower doses. It is possible that the reduction in inhibitory interneurons seen in this anatomical study would be less dramatic at lower doses of VPA exposure.

Furthermore, it is unknown if these changes in parvalbumin are due to VPA directly acting on neurogenesis in the superior colliculus or whether this teratogen acts to effect the expression of parvalbumin alone. To date, no studies probing VPA's developmental affects, if any, on the colliculus have been published.

\section{MECHANISMS OF VPA TERATOGENICITY}

VPA can act through several biochemical mechanisms. It is unknown which of these is responsible for the teratogenic consequences, or whether these are due to a yet to be defined mechanism. The two most extensively studied mechanisms by which VPA exerts its actions are as a histone deacetylase I (HDAC) inhibitor and as a GABA transaminase inhibitor. As an HDAC I inhibitor, VPA exerts epigenetic regulation on cellular development, affecting temporal and spatial control of gene activity. Exposure to such an insult during prenatal development, a peak time for cell proliferation and differentiation, is likely to have dire consequences.

VPA is used as an antiseizure agent due to its role as a GABA transaminase inhibitor: it suppresses the catabolism of GABA and therefore leads to an increase in GABA levels. This immediate effect may be detrimental to the embryo in at least two ways. First, because GABA acts as an excitatory neurotransmitter in the embryo (Ben-Ari, 2002; Owens and Kriegstein, 2002), an increase in GABA concentrations might be excitotoxic (Lombardo et al., 2005; Manent et al., 2007; Levav-Rabkin et al., 2010). Second, because GABA plays a critical role in guiding embryonic neuronal migration and development (Loscher, 1982; Leret et al., 2007), a disruption in GABA concentration gradients is likely to interfere with the development of the CNS. Both of these mechanisms might contribute to the anatomical and behavioral deficits reported here.

A transient surge in GABA during embryogenesis can result in chronic down-regulation of GAD (glutamic acid decarboxylase), the rate-limiting enzyme in the synthesis of GABA. This chronic effect - likely affected through VPA's role as a histone deacetylase inhibitor (Phiel et al., 2001) - can result in a reduction in GABA that persists into adulthood (Leret et al., 2007).

Thus, VPA's effects on the structure of the superior colliculus and other affected structures - likely involve both immediate and lasting effects on GABA metabolism. In fact, the acute and chronic effects of VPA on GABA levels, and the ubiquitous distribution of GABA and its receptors in the nervous system, suggest that embryonic exposure to VPA will likely affect a number of brain structures. Indeed, a single injection of VPA at E12.5 - a paradigm used in the present study - has been previously shown to result in abnormalities in a number of CNS regions including the brainstem, the cerebellum, the neocortex, and the amygdala (Rodier et al., 1996; Ingram et al., 2000; Markram et al., 2008; Rinaldi et al., 2008a). Here we demonstrate that VPA alters the anatomy of the superior colliculus, as well as colliculus-dependent behaviors.

\section{RELEVANCE TO HUMAN DISEASE}

Many disorders are associated with abnormal sensory processing. For example, patients with fetal valproate syndrome, autism spectrum disorders, attention deficit/hyperactivity disorders and putative sensory processing disorders suffer from sensory processing deficits (Mangeot et al., 2001; Baranek et al., 2006, 2007). Indeed some researchers consider prenatal VPA exposure as a relevant model for autism (Rodier et al., 1996; Schneider and Przewlocki, 2005; Wagner et al., 2006; Markram et al., 2007). We propose that VPA disrupts circuitry involved in sensory processing, a pervasive detriment whose effects encompass multiple disorders.

In fact, it has been suggested that the behavioral manifestations of the disorders mentioned above may be due to an underlying inability to properly process sensory information. A. Jean Ayres, an occupational therapist and developmental psychologist, founded sensory integration theory, which seeks to make a connection between deficits in sensory processing and behavioral problems in school-aged children. She introduced sensory integration therapy as a treatment for such disturbances in sensory processing (Ayres, 1979). Markram et al. (2007), based on their findings on VPA's effects on CNS development, introduced the concept of "Intense World Syndrome" in which hyper-reactivity and hyper-plasticity in the brain's microcircuits is causally responsible for the sensory deficits underlying conditions such as autism. Our behavioral and anatomical findings are consistent with these hypotheses, and indicate that the superior colliculus, a structure that plays a key role in sensorimotor integration, might be involved in the pathogenesis of these disorders of sensory dysfunction.

\section{ACKNOWLEDGMENTS}

This research was funded by PHS grants R01-NS051788 and R01-NS35360. 


\section{REFERENCES}

Alsdorf, R., and Wyszynski, D. F. (2005). Teratogenicity of sodium valproate. Expert Opin. Drug. Saf. 4, 345-353.

Ardinger, H. H., Atkin, J. F., Blackston, R. D., Elsas, L. J., Clarren, S. K., Livingstone, S., Flannery, D. B., Pellock, J. M., Harrod, M. J., Lammer, E. J., Majewski, F., Schinzel, A., Toriello, H. V., Hanson, J. W., Optiz, J. M. and Reynolds, J. F. (1988). Verification of the fetal valproate syndrome phenotype. Am. J. Med. Genet. 29, 171-185.

Arndt, T. L., Stodgell, C. J., and Rodier, P. M. (2005). The teratology of autism. Int. J. Dev. Neurosci. 23, 189-199.

Arpino, C., Brescianini, S., Robert, E., Castilla, E. E., Cocchi, G., Cornel, M. C., de Vigan, C., Lancaster, P. A., Merlob, P., Sumiyoshi, Y., Zampino, G., Renzi, C., Rosano, A., and Mastroiacovo, P. (2000). Teratogenic effects of antiepileptic drugs: use of an International Database on Malformations and Drug Exposure (MADRE). Epilepsia 41, 1436-1443.

Ayres, A. J. (1979). Sensory Integration and the Child. Los Angeles, CA: Western Psychological Services.

Baranek, G. T., Boyd, B. A., Poe, M. D., David, F. J., and Watson, L. R. (2007). Hyperresponsive sensory patterns in young children with autism, developmental delay, and typical development. Am. J. Ment. Retard. 112, 233-245.

Baranek, G. T., David, F. J., Poe, M. D., Stone, W. L., and Watson, L. R. (2006). Sensory experiences questionnaire: discriminating sensory features in young children with autism, developmental delays, and typical development. J. Child. Psychol. Psychiatry 47, 591-601.

Bar-Shalita, T., Vatine, J. J., Seltzer, Z., and Parush, S. (2009). Psychophysical correlates in children with sensory modulation disorder (SMD). Physiol. Behav. 98, 631-639.

Ben-Ari, Y. (2002). Excitatory actions of gaba during development: the nature of the nurture. Nat. Rev. Neurosci. 3, 728-739.

Binkerd, P. E., Rowland, J. M., Nau, H., and Hendrickx, A. G. (1988). Evaluation of valproic acid (VPA) developmental toxicity and pharmacokinetics in Sprague-Dawley rats. Fundam. Appl. Toxicol. 11, 485-493.

Crawley, J. N. (2004). Designing mouse behavioral tasks relevant to autisticlike behaviors. Ment. Retard Dev. Disabil. Res. Rev. 10, 248-258.

Fendt, M. (1999). Enhancement of prepulse inhibition after blockade of GABA activity within the superior colliculus. Brain Res. 833, 81-85.

Fendt, M., Li, L., and Yeomans, J. S. (2001). Brain stem circuits mediat- ing prepulse inhibition of the startle reflex. Psychopharmacology (Berl.) 156, 216-224.

Gruber, A. J., Calhoon, G. G., Shusterman, I., Schoenbaum, G., Roesch, M. R., and O'Donnell, P. (2010). More is less: a disinhibited prefrontal cortex impairs cognitive flexibility. $J$. Neurosci. 30, 17102-17110.

Hargreaves, K., Dubner, R., Brown, F., Flores, C., and Joris, J. (1988). A new and sensitive method for measuring thermal nociception in cutaneous hyperalgesia. Pain 32, 77-88.

Ingram, J. L., Peckham, S. M., Tisdale, B., and Rodier, P. M. (2000). Prenatal exposure of rats to valproic acid reproduces the cerebellar anomalies associated with autism. Neurotoxicol. Teratol. 22, 319-324.

Leret, M. L., Lecumberri, M., GarciaMontojo, M., and Gonzalez, J. C. (2007). Role of maternal corticosterone in the development and maturation of the aminoacidergic systems of the rat brain. Int. J. Dev. Neurosci. 25, 465-471.

Levav-Rabkin, T., Melamed, O., Clarke, G., Farber, M., Cryan, J. F., Dinan, T. G., Grossman, Y., and Golan, H. M. (2010). A sensitive period of mice inhibitory system to neonatal GABA enhancement by vigabatrin is brain region dependent. Neuropsychopharmacology 35, 1138-1154.

Lipton, J. W., Robie, H. C., Ling, Z., Weese-Mayer, D. E., and Carvey, P. M. (1998). The magnitude of brain dopamine depletion from prenatal cocaine exposure is a function of uterine position. Neurotoxicol. Teratol. 20, 373-382.

Lombardo, S. A., Leanza, G., Meli, C., Lombardo, M. E., Mazzone, L., Vincenti, I., and Cioni, M. (2005). Maternal exposure to the antiepileptic drug vigabatrin affects postnatal development in the rat. Neurol. Sci. 26, 89-94.

Loscher, W. (1982). Anticonvulsant and biochemical effects of inhibitors of GABA aminotransferase and valproic acid during subchronic treatment in mice. Biochem. Pharmacol. 31, 837-842.

Manent, J. B., Jorquera, I., Mazzucchelli, I., Depaulis, A., Perucca, E., Ben-Ari, Y., and Represa, A. (2007). Fetal exposure to GABAacting antiepileptic drugs generates hippocampal and cortical dysplasias. Epilepsia 48, 684-693.

Mangeot, S. D., Miller, L. J., McIntosh, D. N., McGrath-Clarke, J., Simon, J., Hagerman, R. J., and Goldson, E. (2001). Sensory modulation dysfunction in children with attention-deficit-hyperactivity disorder. Dev. Med. Child Neurol. 43, 399-406.
Markram, H., Rinaldi, T., and Mark, K. (2007). The intense world syndrome - an alternative hypothesis for autism. Front. Nerusosci. 1:77-96. doi:10.3389/neuro.01.1.1.006.2007

Markram, K., and Markram, H. (2010). The intense world theory - a unifying theory of the neurobiology of autism. Front. Hum. Neurosci. 4:224. doi: 10.3389/fnhum.2010.00224

Markram, K., Rinaldi, T., La Mendola, D., Sandi, C., and Markram, H. (2008). Abnormal fear conditioning and amygdala processing in an animal model of autism. Neuropsychopharmacology 33, 901-912.

Monyer, H., and Markram, H. (2004). Interneuron diversity series: molecular and genetic tools to study GABAergic interneuron diversity and function. Trends Neurosci. 27 90-97.

Moore, S. J., Turnpenny, P., Quinn, A., Glover, S., Lloyd, D. J., Montgomery, T., and Dean, J. C. (2000). A clinical study of 57 children with fetal anticonvulsant syndromes. J. Med. Genet. 37, 489-497.

Owens, D. F., and Kriegstein, A. R. (2002). Is there more to GABA than synaptic inhibition? Nat. Rev. Neurosci. 3, 715-727.

Paxinos, G., and Watson, C. (1998). The Rat Brain in Stereotaxic Coordinates. San Diego: Academic Press.

Phiel, C. J., Zhang, F., Huang, E. Y., Guenther, M. G., Lazar, M. A., and Klein, P. S. (2001). Histone deacetylase is a direct target of valproic acid, a potent anticonvulsant, mood stabilizer, and teratogen. J. Biol. Chem. 276, 36734-36741.

Redgrave, P., Simkins, M., McHaffie, J. G., and Stein, B. E. (1996). Nociceptive neurones in rat superior colliculus. II. Effects of lesions to the contralateral descending output pathway on nocifensive behaviours. Exp. Brain Res. 109, 197-208.

Rinaldi, T., Kulangara, K., Antoniello, K. and Markram, H. (2007). Elevated NMDA receptor levels and enhanced postsynaptic long-term potentiation induced by prenatal exposure to valproic acid. Proc. Natl. Acad. Sci. U.S.A. 104, 13501-13506.

Rinaldi, T., Perrodin, C., and Markram, H. (2008a). Hyper-connectivity and hyper-plasticity in the medial prefrontal cortex in the valproic acid animal model of autism. Front. Neural Circuits 2:4. doi:10.3389/neuro.04.004.2008

Rinaldi, T., Silberberg, G., and Markram, H. (2008b). Hyperconnectivity of local neocortical microcircuitry induced by prenatal exposure to valproic acid. Cereb. Cortex 18, 763-770.
Rodier, P. M., Ingram, J. L., Tisdale, B., and Croog, V. J. (1997). Linking etiologies in humans and animal models: studies of autism. Reprod. Toxicol. 11, 417-422.

Rodier, P. M., Ingram, J. L., Tisdale, B., Nelson, S., and Romano, J. (1996). Embryological origin for autism: developmental anomalies of the cranial nerve motor nuclei. J. Comp. Neurol. 370, 247-261.

Rogers, S. J., and Ozonoff, S. (2005). Annotation: what do we know about sensory dysfunction in autism? A critical review of the empirical evidence. J. Child. Psychol. Psychiatry 46, 1255-1268.

Schneider, T., Labuz, D., and Przewlocki, R. (2001). Nociceptive changes in rats after prenatal exposure to valproic acid. Pol. J. Pharmacol. 53, 531-534.

Schneider, T., and Przewlocki, R. (2005). Behavioral alterations in rats prenatally exposed to valproic acid: animal model of autism. Neuropsychopharmacology 30, 80-89.

Schneider, T., Turczak, J., and Przewlocki, R. (2006). Environmental enrichment reverses behavioral alterations in rats prenatally exposed to valproic acid: issues for a therapeutic approach in autism. Neuropsychopharmacology 31, 36-46.

Stein, B. E., and Meredith, M. A. (1993). The Merging of the Senses. Cambridge: MIT Press.

Stodgell, C. J., Ingram, J. L., O’Bara, M., Tisdale, B. K., Nau, H., and Rodier, P. M. (2006). Induction of the homeotic gene Hoxal through valproic acid's teratogenic mechanism of action. Neurotoxicol. Teratol. 28, 617-624.

Truitt, W. A., Sajdyk, T. J., Dietrich, A. D., Oberlin, B., McDougle, C. J., and Shekhar, A. (2007). From anxiety to autism: spectrum of abnormal social behaviors modeled by progressive disruption of inhibitory neuronal function in the basolateral amygdala in Wistar rats. Psychopharmacology (Berl.) 191, 107-118.

Vorhees, C. V. (1987). Behavioral teratogenicity of valproic acid: selective effects on behavior after prenatal exposure to rats. Psychopharmacology (Berl.) 92, 173-179.

Wagner, G. C., Reuhl, K. R., Cheh, M., McRae, P., and Halladay, A. K. (2006). A new neurobehavioral model of autism in mice: pre- and postnatal exposure to sodium valproate. J. Autism Dev. Disord. 36, 779-793.

Won, H., Mah, W., Kim, E., Kim, J. W., Hahm, E. K., Kim, M. H., Cho, S., Kim, J., Jang, H., Cho, S. C., Kim, 
B. N., Shin, M. S., Seo, J., Jeong, J., Choi, S. Y., Kim, D., Kang, C., and Kim, E. (2011). GIT1 is associated with ADHD in humans and ADHDlike behaviors in mice. Nat. Med. 17, 566-572.

Wyszynski, D. F., Nambisan, M., Surve, T., Alsdorf, R. M., Smith, C. R., and Holmes, L. B. (2005). Increased rate of major malformations in offspring exposed to valproate during pregnancy. Neurology 64, 961-965.

Yeomans, J. S., Lee, J., Yeomans, M. H., Steidl, S., and Li, L. (2006). Midbrain pathways for prepulse inhibition and startle activation in rat. Neuroscience 142, 921-929.

Conflict of Interest Statement: The authors declare that the research was conducted in the absence of any commercial or financial relationships that could be construed as a potential conflict of interest.

Received: 02 June 2011; accepted: 06 October 2011; published online: 20 October 2011.

Citation: Dendrinos G, Hemelt $M$ and Keller A (2011) Prenatal VPA exposure and changes in sensory processing by the superior colliculus.
Front. Integr. Neurosci. 5:68. doi: 10.3389/fnint.2011.00068

Copyright (C) 2011 Dendrinos, Hemelt and Keller. This is an open-access article subject to a non-exclusive license between the authors and Frontiers Media $S A$, which permits use, distribution and reproduction in other forums, provided the original authors and source are credited and other Frontiers conditions are complied with. 